\title{
Lexikalische Kompetenz - ein Plädoyer für eine breitere Auffassung des Begriffs
}

\section{Lexical competence: a plea for a broader definition}

\begin{abstract}
The subject of the present paper is the notion of 'lexical competence'. This type of competence is often analysed in a too narrow sense, although over the years the notion has been broadened. Since in the Author's opinion the definition of lexical competence should include not only the knowledge about vocabulary or its usage, but also the ability to compensate for lexical gaps, a new, slightly broader definition has been formulated and the factors were presented which may influence its development
\end{abstract}

KEYWORDS: lexical competence, vocabulary competence

\section{EINLEITUNG}

Jede Sprache besteht aus Wörtern und grammatischen Regeln, nach denen die Wörter nicht nur verbunden werden, sondern auch eine bestimmte Bedeutung bekommen bzw. ihre aktuelle Bedeutung spezifiziert wird. Ohne Wortschatzkenntnisse sind Fremdsprachenerwerb und -lernen sowie die Verständigung in dieser Sprache völlig unmöglich. Reicht es aber einzelne Wörter zu kennen, damit die Kommunikation erfolgreich wird? Bestimmt nicht. Wortschatzkenntnisse bilden nur eine Grundlage für die Fähigkeit, mit dem Wortschatz richtig umzugehen. Seit der kommunikativen Wende ist klar, dass beim Fremdsprachenlernen nicht Sprachwissen, sondern Sprachfertigkeiten bzw. Kompetenzen eine viel wichtigere Rolle spielen. Daraus ist zu schlussfolgern, dass nicht Wortschatzkenntnisse, sondern die Entfaltung der lexikalischen Kompetenz bei den Lernenden eines der Hauptziele des Fremdsprachenunterrichts darstellen sollte. Was bedeutet aber dieser Begriff? Ist diese Bezeichnung nur mit dem Verstehen der Bedeutung einer lexikalischen Einheit (weiter LE) oder nur mit deren richtiger 
Anwendung gleichzusetzen? In diesem Artikel wird davon ausgegangen, dass der Begriff "lexikalische Kompetenz" oder "Wortschatzkompetenz" sehr oft zu eng bzw. zu einseitig aufgefasst wird. Es wird an dieser Stelle auf zahlreiche Faktoren hingewiesen, die unter der lexikalischen Kompetenz subsumiert werden können bzw. sollen. Bevor eingehender auf den Terminus "lexikalische Kompetenz" eingegangen wird, schenken wird unsere Aufmerksamkeit dem Wortschatzbegriff (weil ein gutes Verständnis dieses Terminus bei der richtigen Auffassung der lexikalischen Kompetenz hilfreich sein kann) sowie dem Ziel der Wortschatzarbeit.

\section{WORTSCHATZ UND WORTSCHATZARBEIT}

Über die Bedeutung des Begriffs Wortschatz ist man sich seit längerer Zeit im Klaren. Wortschatz stellt keine Anzahl von separaten Einzelwörtern dar, weswegen er von dem Begriff Vokabel abgegrenzt werden sollte. Er besteht nicht aus losen Vokabeln, sondern stellt ein System von unterschiedlichen Beziehungen und Verflechtungen dar, die nicht nur das Behalten fördern, sondern auch das schnelle Abrufen, d.h. eine rasche Verfügbarkeit des nötigen Vokabulars ermöglichen. (Beile 1988: 5) Es steht außer Zweifel, dass lexikalisches Wissen nicht nur bei der Grammatikarbeit, sondern auch bei jeder Sprachfertigkeit, d.h. beim Hörverstehen, Leseverstehen, Schreiben und Sprechen oder bei der Sprachmittlung eingesetzt wird, weil der Lernende bei jeder sprachlichen Aktivität mit dem Wortschatz einer Sprache konfrontiert wird und von ihm nicht nur bei der Produktion Gebrauch macht, sondern ihn auch zum Enkodieren bei der Rezeption benutzt. Wortschatzarbeit (weiter WA) ist also sehr eng mit der Arbeit an allen Sprachfertigkeiten verbunden. Daraus resultiert das Ziel der WA

Wenn man vom Ziel der Wortschatzarbeit spricht, bezieht man sich meistens in der einschlägigen Literatur auf zwei Definitionen. Erstens handelt es sich hier um die Feststellung von Desselmann und Hellmich (1986: 146), deren Meinung nach das Ziel der WA „in der Aneignung eines dauerhaften, schnell abrufbaren, disponibel verknüpfbaren und korrekt anwendbaren Wortschatzbesitzes [liegt], der auf die Realisierung von relevanten Kommunikationsabsichten und Bewältigung bestimmter Themen und Kommunikationssituationen abgestimmt ist." Eine ähnliche Formulierung dieses Ziels finden wir bei Löschmann (1993: 29), der Folgendes feststellt: „Ziel der WSA [Wortschatzarbeit] ist die Aneignung eines intentions- und situationsgerecht anwendbaren, dauerhaften, schnell abrufbaren, disponibel verknüpfbaren sowie normgerecht verwendbaren Wortschatzbesitzes." Die beiden einander recht ähnlichen Formulierungen bezüglich des Ziels der WA betonen den 
Gebrauchsaspekt einer LE und verweisen darauf, dass die Arbeit an einem neuen, dem bereits kennen gelernten sowie schon dauerhaft gespeicherten Vokabular dazu führen sollte, dass die Lernenden zum einen die Lexik speichern. Zum anderen geht es um die Befähigung des Lernenden dazu, den recht neuen Wortschatz situationsadäquat gebrauchen zu können. Löschmann (1993: 23) betont auch, dass von Qualität des Wortschatzbesitzes (also einer gut entwickelten lexikalischen Kompetenz) gesprochen werden kann, wenn der Lernende den Wortschatz zwar ziel-, gegenstands-, und bedingungsadäquat verwenden kann, aber besonders wenn er dabei früher nicht bewältigte Kommunikationssituationen dank seinen schöpferischen Lösungen meistern kann.

\section{ZUM BEGRIFF LEXIKALISCHE KOMPETENZ}

Bevor man zur Klärung des Begriffs lexikalische Kompetenz bzw. Wortschatzkompetenz übergeht, sind Überlegungen darüber anzustellen, was es eigentlich bedeutet ein Wort zu kennen. Die Antwort auf diese Frage ist nicht immer gleich, weil wie Aguado (2004: 237) feststellt, sie u. a. auch vom Sprachstand der Lernenden abhängt. Wenn man von der lexikalischen Kompetenz eines Anfängers spreche, dann könne sich diese darin äußern, dass der Lernende das Wort verstehe oder es übersetzen könne. Im Falle der fortgeschrittenen Lernenden, kommen noch weitere Faktoren hinzu. In diesem Sinne wird im folgenden Artikel von der lexikalischen Kompetenz der Nicht-Anfänger gesprochen.

Obwohl der Stellenwert und Bedeutung der lexikalischen Kompetenz unbestritten ist, besteht keine Einigkeit darüber, zu welchen Oberfertigkeiten sie gehört. Auf der einen Seite sieht Löschmann (1993: 142) die lexikalische Kompetenz als einen Teil der sprachlich-kommunikativen Kompetenz. Auf der anderen Seite wird im GER (2001: 110ff.) die lexikalische Kompetenz neben der grammatischen, semantischen und phonologischen Kompetenz als eine der vier Komponenten der linguistischen Kompetenz aufgefasst, die einen Teil der Kommunikativen Sprachkompetenz darstellt.

In der fremdsprachendidaktischen Literatur wird manchmal die lexikalische Kompetenz nur ganz allgemein definiert. Einige Definitionen erklären diesen Begriff als „Wissen und Können“ (Król 2008: 48) oder folgendermaßen "Sie umfasst die Kenntnis des Vokabulars einer Sprache, das aus lexikalischen und aus grammatischen Elementen besteht, sowie die Fähigkeit, es zu verwenden“ (GER 2001: 111). Bei dieser zweiten Formulierung scheinen zwei Sachen ein bisschen unpräzise zu sein. Erstens wird beim Gebrauchsaspekt nicht angegeben, dass zur lexikalischen Kompetenz auch 
die Fähigkeit gehört, mit Wortschatzlücken, mit schlecht oder nicht korrekt internalisierten Wortbeständen richtig umgehen zu können. Dies wird erst bei der weiteren Beschreibung des Wortschatzspektrums auf bestimmten Niveaustufen präzisiert, weil dort indirekt auf Kompensationsstrategien verwiesen wird. (GER 2001: 112) Der zweite Problembereich der Auffassung von lexikalischer Kompetenz ist deren eingeschränktes Verständnis, weil im GER (2001: 116) die Semantik von der lexikalischen Kompetenz ausgegrenzt wird. Hier wird die semantische Kompetenz als eine separate Kompetenz aufgefasst, die sozusagen neben der lexikalischen und grammatischen Kompetenz als Bestandteil der linguistischen Kompetenz stehe. Diese wird als „Fähigkeit Lernender, sich der Organisation von Bedeutung bewusst zu sein und diese zu kontrollieren" verstanden. (ebenda) Dieser Einteilung liegt folgende Ausgangsposition zugrunde: „Sprachen beruhen bekanntlich auf einer Organisation der Form und einer Organisation der Beziehung. Die Kategorien beider Organisationen stehen in meist zufälliger Bedeutung zueinander." Ähnlich ist es mit phonologischer und orthographischer Kompetenz, die nicht der lexikalischen, sondern der linguistischen Kompetenz untergeordnet sind und sozusagen neben der lexikalischen Kompetenz „existieren“. (GER 2001: 116) Dies kann ein bisschen verwunderlich erscheinen, weil sich doch die grafische, bzw. phonetische Kompetenz auf Wörter beziehen, weswegen meiner Ansicht nach die Aussonderung der beiden Kompetenzarten keinen großen Sinn hat. Darüber hinaus soll meines Erachtens die Kenntnis der lexikalischen Bedeutung zur lexikalischen Kompetenz gehören, weil ich die Bedeutung, also die semantische Kompetenz als einen integralen Bestandteil der Wortschatzkompetenz verstehe und von deren Ausklammerung nicht überzeugt bin.

Wie soll demnach die lexikalische Kompetenz verstanden werden? Grundlagen für die Erforschung dieses Begriffs legte Richards (1976 nach Aguado 2004: 237), der eine zwar umfangreiche, dennoch nicht erschöpfende Liste von vielen Komponenten der lexikalischen Kompetenz formulierte. Diese bezogen sich auf verschiedene Aspekte des Wortschatzes, d.h. auf die Semantik, Morphologie und Syntax. Für ihn äußerte sich die Kenntnis eines Wortes darin:

- man kennt den semantischen Wert einer LE,

- man kennt verschiedene Bedeutungen eines Wortes,

- man kennt ein Netzwerk von Assoziationen zwischen dem Wort und anderen Wörtern,

- man kennt die Grenzen des Gebrauchs einer LE,

- man kennt die Grundform, sowie Ableitungen eines Wortes,

- man kennt das syntaktische Verhalten einer LE,

- man kennt die kombinatorische Komponente eines Wortes. 
Zwar bildet immer das Wissen in einem Bereich die Grundlage zur Entfaltung einer Kompetenz, aber es soll nur als Ausgangspunkt zu deren Entwicklung aufgefasst werden. Aus diesem Grunde kann man einige Schwachpunkte der oben angeführten Definition bemerken, weil diese eigentlich nur die deklarative Ebene berücksichtigt, wobei solche wichtigen Faktoren wie der Verwendungsaspekt und die prozedurale Ebene außer Acht gelassen wurden. Bei der lexikalischen Kompetenz ist nicht nur die rezeptive Kenntnis des Vokabulars von Bedeutung, sondern auch die produktive Ebene. Es ist bestimmt einfacher ein Wort wieder zu erkennen als es in einem entsprechenden Moment abzurufen und zu gebrauchen. Wichtiger ist also die Fähigkeit, eine LE selbstständig und aktiv, adäquat und sprachnormgerecht gebrauchen zu können. (Aguado 2004: 237)

Die weiteren Definitionsversuche haben den Verwendungsaspekt der LE schon berücksichtigt. Ein Beispiel dafür kann Wallace (1982 nach Rzewólska 2008: 40) sein, der in Anlehnung an die Arbeit von Richards (1976) auch die Komponenten der Kenntnis einer LE formulierte. Diese äußert sich laut ihm darin, dass der Lernende (bzw. Muttersprachler):

- die LE in der schriftlichen Form oder der mündlichen Realisierung erkennen kann,

- die LE in einem bestimmten Moment reproduzieren kann,

- die LE u.a. mit einem Gegenstand assoziieren kann,

- die LE korrekt (auch im mündlichen Gebrauch) einsetzen kann,

- die LE korrekt aufschreiben kann,

- die LE in korrekten Verbindungen (Kollokationen) gebrauchen kann,

- die LE auf einer korrekten stilistischen Ebene anwenden kann,

- sich der mit der LE verbundenen Konnotationen und Assoziationen bewusst ist.

Der Verwendungsaspekt einer LE wurde auch bei der Erklärung dieses Terminus von Nation (1990, 2001 nach Aguado 2004: 237f) miteinbezogen, weil er schon von drei Aspekten spricht, die unter die lexikalische Kompetenz subsumiert werden können. Außer den zwei von Richards (1976) genannten, nämlich Form und Bedeutung, berücksichtigt er noch den Gebrauchsaspekt, also die Fähigkeit, ein Wort korrekt im Kontext sowie pragmatisch angemessen verwenden zu können.

Die nächsten Wissenschafter, die den Begriff Wortschatzkompetenz zu ihrem Forschungsgegenstand machten, waren Trèville und Duquette (1996: 98 nach Rzewólska 2008: 40), die folgende Komponenten der lexikalischen Kompetenz formulierten:

- die sprachliche Komponente (umfasst das, was Richards 1976 formulierte, also Kenntnis der Bedeutung aber auch der Schreibweise, der morphosyntaktischen Geprägtheit der LE), 
- die diskursive Komponente,

- die referentielle Komponente,

- die soziokulturelle Komponente (u.a. Kenntnis des Registers, Kenntnis der kulturellen Geprägtheit der LE),

- die strategische Komponente (die Fähigkeit mit unzureichendem Wortschatzbestand umzugehen, Kommunikationssituationen $\mathrm{zu}$ meistern und Wortschatzlücken zu kompensieren).

Eine Neuerung bei dieser Erklärung des Begriffs war der Hinweis auf die kulturelle Komponente sowie auf die strategische Komponente, die beim richtigen Umgang mit der fremdsprachigen Lexik von Bedeutung sind.

Man könnte gegen die Definitionen von Wortschatzkompetenz einwenden, dass sie über die Lexik hinausgehen und auch teilweise die Grammatik, bzw. grammatische Kompetenz mit einschließen. Viele Wissenschaftler sind jedoch von der Untrennbarkeit von Wortschatz und Grammatik überzeugt (vgl. Aguado 2004, Król 2001). Häusermann und Piepho (1996: 81) behaupten sogar, dass die Grammatik nur die Beobachtung dessen ist, wie sich der Wortschatz verhält und behaupten, dass Wortschatz und Grammatik nur „verschiedene Dimensionen eines Systems darstellen“. Jedes Wort ist nämlich schon grammatisch vorgeprägt, d.h. es gehört zu einer Wortart, hat eine bestimmte Valenz und steht in bestimmten syntagmatischen bzw. paradigmatischen Relationen, die beim Gebrauch eines Wortes im Satz bzw. in einer Äußerung immer berücksichtigt werden müssen. Der korrekte Wortschatzgebrauch bedarf des grammatischen Wissens und dessen bewussten Einsatzes.

Die Bedeutung des Begriffs lexikalische Kompetenz muss jedoch über das deklarative Wissen hinausgehen und den Zugang zum Wortschatzwissen umfassen. Über Wortschatzkompetenz zu verfügen bedeutet auch, ein bestimmtes Wort in einem bestimmten Moment, in einer kommunikativen Situation abrufen und anwenden zu können und bei unzureichenden Wortschatzbeständen, die Lücken in der Wortschatzkenntnis kompensieren zu können. Da lexikalische Kompetenz einerseits als rezeptive Wortschatzkompetenz verstanden wird, andererseits das oberste Ziel der Wortschatzarbeit gerade die produktive Kompetenz darstellt, muss in Bezug auf die beiden der Begriff um strategische Fähigkeiten ergänzt werden. Dies bedeutet, dass zur lexikalischen Kompetenz einerseits die Fähigkeit gehört, die Bedeutung eines unbekannten Wortes aus dem Kontext zu erschließen. Andererseits muss der kreative Umgang mit ungenügendem Wortschatzwissen in der Produktion auch unter die lexikalische Kompetenz subsumiert werden. Daraus ist zu schlussfolgern, dass die nächste Komponente der lexikalischen Kompetenz die Kenntnis der Kompensationsstrategien sowie deren sprachadäquater Einsatz darstellen sollte. (Aguado 2004: 238, 247) 
Im Folgenden wird für eine breit gefächerte Auffassung der Wortschatzkompetenz plädiert. Da Wortschatzkompetenz sowohl die rezeptive als auch die produktive Ebene des Wortschatzerwerbs, den potenziellen Wortschatz sowie den angemessenen und adäquaten Umgang mit Unvollkommenheit des Wortschatzbesitzes umfassen sollte, sind all diese Ebenen in der Begriffsbestimmung zu berücksichtigen. Worin äußert sich die lexikalische Kompetenz? Diese zeigt sich zwar im Allgemeinen in der Kenntnis einer lexikalischen Einheit, obwohl wie Rzewólska (2008: 39) feststellt, man nie oder sehr selten den ganzen Umfang eines Wortes kennt. Man habe nur ein oberflächliches Wissen über dessen Bedeutung, das sich in der Kommunikation als ausreichend erweist. Die Wortschatzkompetenz kommt aber auch in der Bewältigung einer kommunikativen Situation zum Ausdruck.

Die breitere Auffassung der Wortschatzkompetenz müsste meines Erachtens mehr Komponenten enthalten. Diese äußert sich meiner Ansicht nach auf der Ebene der Rezeption (dabei wird auch der potenzielle Wortschatz miteinbezogen) in folgenden Teilkompetenzen des Lernenden. Er kann:

- die LE in der schriftlichen und mündlichen Realisierung erkennen,

- unter verschiedenen Bedeutungen eines Wortes die in einer bestimmten Situation entsprechende Bedeutung erkennen, d.h. der akustischen bzw. graphischen Form die richtige Bedeutung zuordnen,

- die Bedeutung eines zusammengesetzten Wortes anhand bekannter Lexeme erschließen,

- auf Grund der bekannten lexikalischen Einheiten aus dem Kontext die Bedeutung einer unbekannten LE erschließen.

Darüber hinaus soll sich der Fremdsprachenlernende der mit einer LE verbundenen Konnotationen und Assoziationen sowie deren landeskundlich relevanter Aspekte bewusst sein.

Auf der Ebene der Produktion äußert sich die lexikalische Kompetenz in folgenden Teilkompetenzen. Der Lernende:

- kann ein passendes Wort sprachlich korrekt gebrauchen (korrekte Schreibweise, Aussprache),

- kann unter vielen Bedeutungen des Wortes ein dem Kontext entsprechendes Wort auswählen,

- kennt das syntaktische Verhalten der LE und kann diese korrekt in Bezug auf Morphosyntax gebrauchen (dabei hilft die Kenntnis des syntaktischen Wertes, Kenntnis der Valenz, Kenntnis von Genus),

- kann eine bestimmte LE situationsadäquat gebrauchen (Pragmatik),

- kennt die kombinatorische Komponente eines Wortes (Kenntnis der kollokativen Verbindungen) und kann eine LE mit entsprechenden LE-en in Verbindung setzen,

- kann ein Wort in einem bestimmten Kontext korrekt einsetzen, 
- kennt die Grenzen eines Wortes und kann dieses auf der bestimmten Stilebene einsetzen,

- kennt ein Netzwerk von Assoziationen zwischen dem Wort und anderen Wörtern und kann zu einem Wort ihm passende Vokabeln abrufen,

- kennt die kulturelle Geprägtheit der LE und die Unterschiede zwischen Umfang des Wortes in der L1 und der Zielsprache,

- kann von der Grundform ein nötiges Wort ableiten (z.B. von Verb ein Substantiv),

- kann ein zusammengesetztes Wort bilden,

- kann bei mangelnden Wortschatzbeständen die Lücken in der Lexik kompensieren (durch Umschreibungen, Paraphrasierungen, Definitionen, bzw. definitionsähnliche Versuche, Einsatz von Antonymie und Synonymie),

- kann anhand des Wortbildungswissens kreativ mit dem Wortschatz umgehen, d.h. für bestimmte kommunikative Situationen für ihn neue Wörter bilden,

- kann anhand der in einem einsprachigen Wörterbuch enthaltenen Informationen ein Wort in dem Kontext richtig gebrauchen,

- kann mit einem zweisprachigen Wörterbuch arbeiten und die neuen dort gefundenen Vokabeln richtig einsetzen,

- kann seinen Wortschatzzuwachs planen, organisieren und überwachen.

Bei der Entfaltung der lexikalischen Kompetenz ist es außerordentlich wichtig, die Lernenden dazu zu befähigen, das Lexikon selbstständig zu erweitern, d.h. Wörter in Wörterbüchern zu finden, diese sprachlich korrekt sowie situationsadäquat einzusetzen und in das mentale Lexikon so $\mathrm{zu}$ „integrieren", damit die Speicherung dauerhaft ist. Damit hängen noch folgende Teilkompetenzen der Wortschatzkompetenz zusammen, nämlich die Fähigkeit der korrekten Wörterbuchbenutzung sowie auch die Kenntnis und der adäquate Einsatz von kognitiven Lernstrategien. Der Fremdsprachenlernende sollte darauf vorbreitet werden, seinen Wortschatz selbstständig und planmäßig zu erweitern und zu vertiefen. Dazu braucht er das Wissen über Speicherung und Behaltensprozesse.

\section{WIE KANN DIE LEXIKALISCHE KOMPETENZ ENTWICKELT WERDEN?}

Aus der oben dargestellten breit ausgelegten Auffassung der lexikalischen Kompetenz ist zu schlussfolgern, dass bei der Entwicklung der Wortschatzkompetenz der Lernende nicht nur auf den korrekten und situationsangemessenen Einsatz des zielsprachigen Wortschatzes vorbereitet werden sollte. Weiterhin geht es hier auch um seine Vorbereitung auf den Umgang 
mit ungenügenden Wortschatzbeständen, wobei einerseits die Herausbildung der Kommunikations- und Kompensationsstrategien, andererseits die Fähigkeit der Benutzung verschiedener Arten von Wörterbüchern hilfreich sein könnten. Wie soll oder kann eine fundierte lexikalische Kompetenz entwickelt, entfaltet bzw. gefördert werden? Die richtig betriebene Wortschatzarbeit kann zweifelsohne den größten Beitrag zur Entfaltung der Wortschatzkompetenz leisten. Schon im schulischen Fremdsprachenunterricht können nämlich die Lernenden entweder den Eindruck gewinnen, dass Wortschatzlernen die Aneignung von losen Vokabeln bedeutet (was natürlich falsch ist) oder zur Ansicht kommen, dass Wortschatz eine Verflechtung von bestimmten Vokabeln darstellt und der Einsatz einer Vokabel oft den Gebrauch einer konkreten LE erfordert. Bei der richtigen Vorgehensweise können die Lernenden von Anfang an auf den richtigen Wortschatzerwerb vorbereitet werden. Da wie Löschmann (1993: 25) feststellt, lexikalische Einheiten in ihrer Mehrdimensionalität begriffen werden sollten, ist es unabdingbar, jede LE mit allen zu ihr gehörenden Komponenten (und zwar sowohl in Bezug auf die Bedeutung sowie auch die Form) auf der einen Seite einzuführen auf der anderen zu speichern.

Von großer Bedeutung ist auch der ganze Übungsvorgang, d.h. systematisches Üben, das nach bestimmten Prinzipien aufgebaut werden sollte. Dabei ist davon auszugehen, dass sich Wortschatzübungen zum Ziel setzen, die zur Realisierung der kommunikativen Sprechabsichten dienenden lexikalischen Einheiten nicht nur rezeptiv, sondern auch produktiv verfügbar zu machen. Durch die lexikalischen Übungen soll demnach der Lernende dazu befähigt werden „die vorher eingeprägten Wörter in bestimmten Kommunikationssituationen entweder zum Verstehen oder zum Ausdruck von Sinngehalten aus dem Gedächtnis abzurufen“. (Segermann 1992: 89) Gerade um das späte Abrufen einer bestimmten lexikalischen Einheit in einer Kommunikationssituation zu ermöglichen, sind bestimmte kommunikative Übungen notwendig. Król (2008: 48) schlägt in Bezug auf die Entwicklung der lexikalischen Kompetenz den integrativen Ansatz vor, in dem die WA mit der Entwicklung aller Sprachfertigkeiten, mit der grammatischen Kompetenz und Kontextualität integriert werden sollte. Das nächste zu beachtende Prinzip bezieht sich auf die Steigerung des Schwierigkeitsgrades bei der Entwicklung des rezeptiven sowie des produktiven Wortschatzes. Der Lernende sollte nämlich die Möglichkeit bekommen, seine lexikalische Kompetenz stufenweise aufzubauen. (Segermann 1992: 279)

Das Wie der WA bezieht sich nicht nur auf die Übungsphase, sondern auch auf die Festigungs-, Wiederholungs- und Kontrollphase. Die Wiederholung der LE-en ist zum einen wichtig, weil Wortschatzkenntnisse vergessensanfällig sind. Zum anderen resultiert ihre Wichtigkeit daraus, dass 
man in diesem Prozess nicht nur den Wortschatz reaktiviert, sondern ihn auch viel dimensional im Gedächtnis verankert. Die kommunikative Einbettung der LE sollte auch bei der Wortschatzkontrolle Berücksichtigung finden. Da WA der Entwicklung sprachlich-kommunikativer Kompetenz untegeordnet sein sollte, sollte aus diesem Grunde bei der Wortschatzkontrolle die Kompetenzüberprüfung im Vordergrund stehen. (Löschmann 1993: 109, 144)

Da auf die Entwicklung der Wortschatzkompetenz die Motivation einen großen Einfluss zu haben vermag und man den Wortschatz nicht nur um des Wortschatzes willen einführt, sondern zum Zweck der Kommunikation in der Fremdsprache bzw. zur Realisierung anderer Ziele, sollten die Lernenden die Überzeugung bekommen, dass ihnen der zu lernende Wortschatz nützlich ist. Deshalb ist die Integration der WA mit allen Sprachfertigkeiten so bedeutsam. Von großer Bedeutung ist zweifelsohne auch die Arbeit an produktiven lexikalischen Strategien, die in die WA integriert werden sollte. Dabei können Übungen zum Paraphrasieren behilflich sein, weil Paraphrasierungen, die auch in der Muttersprache von Native Speakers eingesetzt werden, von fortgeschrittenen Fremdsprachenlernern als Kompensationsformen zur Ausgleichung von Wortschatzdefiziten herangezogen werden können. Die nächste Übungsart, die der Herausbildung produktiver lexikalischer Strategien dienen kann, sind Wortbildungsübungen, wobei Zimmermann (1990: 444) auch die verstärkte Arbeit an der produktiven Wortbildung postuliert, weil er den Eindruck gewonnen hat, dass man sich bei der Wortschatzarbeit eher auf die analysierende Wortbildungsdidaktik konzentriert. Empfehlenswert ist jedoch eine andere Vorgehensweise, nämlich der kreative Umgang mit eigenen Wortschatzbeständen, bei dem man die Wortbildungskenntnisse in kommunikativen Aufgaben zur Produktion gebraucht. Dies formuliert Zimmermann (1990: 444) folgendermaßen: „Fortgeschrittene Lerner müssen das Lexikon einer Fremdsprache als ähnlich produktiv und kreativ wie die Syntax erfahren und wissen, dass und wie man bei Bedarf innovative Benennungen bilden kann."

De Florio-Hansen (2004: 85) geht davon aus, dass gerade WA zu den Bereichen gehört, die sich am besten für selbst bestimmtes Lernen eignen, weil WA als ein individueller Prozess aufgefasst werden kann. Viele Lernende entwickeln im Laufe „ihrer sprachlichen Laufbahn“ eine Palette von geeigneten Lerntechniken, bzw. -strategien, mit deren Hilfe sie den Wortschatz selbstständig entwickeln können. Dies sollte jedoch nicht als eine Möglichkeit, sondern als Pflicht der schulischen oder universitären WA aufgefasst werden, weil jeder Fremdsprachenlernende auf seine weitere individuelle Entfaltung der Wortschatzkompetenz vorbereitet werden sollte. Aus diesem Grunde sind im Rahmen der WA die Übungen zum Wörterbuchgebrauch 
nicht wegzudenken. Es handelt sich hier nicht nur um den Gebrauch der zweisprachigen, sondern auch der einsprachigen Wörterbücher. Diese Fähigkeit, das Wörterbuch richtig einsetzen zu können, kann den Lernenden helfen, "sich geeignete Ausschnitte eines Wortschatzes selbst auszuwählen und für das Lernen aufzubereiten“. (ebenda: 85) Die Lerner sollten darüber hinaus darauf vorbreitet werden, dass beim Wortschatzerwerb auch inzidentelles Lernen von LE-en eine Rolle spielen kann, weil sie bei bestimmten Aufgaben feststellen, bzw. entscheiden können, welches Wort ihnen bei Realisierung bestimmter kommunikativer Situationen jetzt oder in der Zukunft nötig sein könnte. Nur bei der breiteren oben dargestellten Auffassung von Wortschatzkompetenz kann der Lernende autonom seine Wortschatzarbeit betreiben.

\section{LITERATURVERZEICHNIS}

Aguado, K., 2004. Evaluation fremdsprachlicher Wortschatzkompetenz: Funktionen, Prinzipien, Charakteristika, Desiderate. In. Fremdsprachen Lehren und Lernen 33, 231-250.

Beile, W., 1988. Übungen und Üben im fremdsprachlichen Lernprozess. In: Der fremdsprachliche Unterricht, Heft 92, 4-7.

Bohn, R., 1999. Probleme der Wortschatzarbeit. München: Langenscheidt.

De Florio-Hansen, I., 2004. Wortschatzerwerb und Wortschatzlernen von Fremdsprachenstudierenden. Erste Ergebnisse einer empirischen Untersuchung. In: Fremdsprachen Lehren und Lernen 33, 83-113.

Desselmann, G. / Hellmich, H., 1986. Didaktik des Fremdsprachenunterrichts (Deutsch als Fremdsprache). Leipzig: Verlag Enzyklopädie.

Häussermann, U. / Piepho, H.E., 1996. Aufgaben-Handbuch. Deutsch als Fremdsprache. Abriß einer Aufgaben- und Übungstypologie. München: iudicium.

Gemeinsamer europäischer Referenzrahmen für Sprachen: lernen, lehren beurteilen. 2001. Europarat. Rat für kulturelle Zusammenarbeit, Berlin / München / Wien / Zürich / New York: Langenscheidt.

Król, A., 2008. Zintegrowany rozwój kompetencji leksykalnej w dydaktyce języka specjalistycznego. In: Przeglad Glottodydaktyczny 25, 47-52.

Löschmann, M., 1993. Effiziente Wortschatzarbeit. Alte und neue Wege. Frankfurt am Main: Peter Lang.

Nation, I.S.P., 1990. Teaching \& Learning Vocabulary. New York: Newbury House.

Nation, I.S.P., 2001. Learning vocabulary in another language. Cambridge: Cambridge University Press.

Richards, J.C., 1976. The role of vocabulary teaching. In: TESOL Quarterly 10, 77-89.

Rzewólska, A., 2008. Kompetencja leksykalna i nauczanie języków obcych. In: Języki Obce w Szkole 1, 38-44.

Segermann, K., 1992. Typologie des fremdsprachlichen Übens. Bochum: Brockmeyer.

Treville, M.C. / Duquette, L., 1996. Enseigner le vocabulaire en classe de langue. Vanves: Hachette.

Wallace, M.J., 1982. Teaching Vocabulary. London: Heinemann Educational Books.

Zimmermann, R., 1990. Lexikalische Strategien: Perspektiven für die Wortschatzarbeit? In: Die Neueren Sprachen 89, 426-452. 
